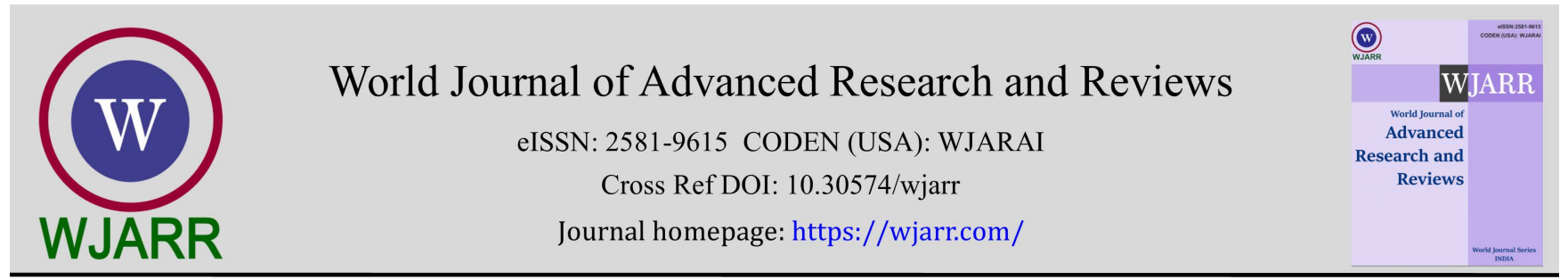

(RESEARCH ARTICLE)

\title{
Peripheral blood abnormalities in patients with Covid 19 hospitalized in the pneumology department of Marrakesh University Hospital
}

\author{
Selma Abdala *, Salma Aitbatahar and Lamyae Amro \\ Faculty of Medicine and Pharmacy, Cadi Ayyad University, Department of Pneumology, ARRAZI Hospital, Mohamed VI \\ University Hospital Center, Marrakesh, Morocco.
}

World Journal of Advanced Research and Reviews, 2021, 09(01), 046-049

Publication history: Received on 23 December 2020; revised on 29 December 2020; accepted on 01 January 2021

Article DOI: https://doi.org/10.30574/wjarr.2021.9.1.0497

\begin{abstract}
In December 2019, Coronavirus 2019 due to SARS CoV 2 was first discovered in Wuhan, China and then rapidly spread to more than 200 countries. Until now, there is no specific vaccine and no effective treatment, so it is very important from a clinical point of view to predict the evolution of the disease. Thus, in order to further analyze the value of the clinical application of blood count parameters in the diagnosis or management of COVID-19 (Coronavirus disease 2019) patients. Peripheral blood abnormalities of 102 COVID-19 patients hospitalized in the pneumology department of CHU Mohamed VI in Marrakesh were analyzed retrospectively and compared to SARS CoV 2 negative patients. Results: WBCs in the patient group were $8.32 \times 109 / \mathrm{L}$ vs $5.85 \times 109 / \mathrm{L}, \mathrm{p}<0.001$, an elevated neutrophil count $(5.33 \times 109 / \mathrm{L}$ vs 3.27 $\times 109 / \mathrm{L}, \mathrm{p}<0.001$ ), a lower hemoglobin concentration (13.5 [10.6-16.37] g/dl vs 14.8 [13.5-15.6] g/dl, p<0.001), and a higher Neutrophils-to-lymphocytes ratio (2.63 vs 1.52 , p<0.001). The severe cases had a higher white blood cell count $(14,279( \pm 6,564)$ vs. $7,471( \pm 2,609) \times 109 / \mathrm{L}, \mathrm{p}<0.001)$, a much higher neutrophil count $(12,547( \pm 6,698)$ vs. 4,468 $( \pm 1,882) \times 109 / \mathrm{L}, \mathrm{p}<0.001)$. Lymphopenia $(0.851( \pm 0.406)$ vs. $2.138( \pm 1.103) \times 109 / \mathrm{L}, \mathrm{p}<0.001)$, NLR (14.74 vs. 2.08) and Platelets-to-lymphocytes ratio (145 vs. 117) were higher than the non-severe COVID-19 cases. Conclusion: In light of these data, we deduce that regular monitoring of blood counts should be involved to judge the progression and prognosis of COVID-19.
\end{abstract}

Keywords: Peripheral blood; SARS CoV 2; Neutrophils; Lymphocytes

\section{Introduction}

In December 2019, Coronavirus 2019 due to SARS coV 2 was first discovered in Wuhan, China and then rapidly spread to more than 200 countries. As of July 1, 2020, according to the World Health Organization (WHO), there were 10,357,662 confirmed cases of Covid-19 and 508,055 deaths. Until now, there is no specific vaccine and no effective treatment, so it is very important from a clinical point of view to predict the evolution of the disease. Previous studies have shown that neutrophil/lymphocyte and platelet/lymphocyte ratios are predictive of infectious disease progression [1,2], a study by Guan et al. of 1099 patients with COVID-19 showed abnormalities in the hematopoietic peripheral blood system of some patients [3]. Thus, in order to further analyze the value of the clinical application of these blood parameters in the diagnosis or management of COVID-19 patients, the peripheral blood abnormalities of 102 COVID-19 patients hospitalized in the pneumology department of CHU Mohamed VI of Marrakech were analyzed retrospectively, which is reported below.

\footnotetext{
* Corresponding author: Selma Abdala

Faculty of Medicine and Pharmacy, Cadi Ayyad University, Department of Pneumology, ARRAZI Hospital, Mohamed VI University Hospital Center, Marrakech, Morocco.
} 


\section{Patients and methods}

\subsection{Diagnostic Criteria}

All Covid-19 cases were confirmed by the use of RT-PCR on nasal swab specimens.

Patients with hemopathy or who received a blood transfusion during hospitalization were excluded from the study.

2.1.1. The COVID-19 cases were divided into two categories according to severity: mild to moderate and severe cases, the latter representing at least one of the criteria listed below

- Neurological Disorders: Disorders of Consciousness

- Polypnea: FR> or equal to 30 cycles per min.

- $\quad$ Systolic BP $<90 \mathrm{mmHg}$

- Heart rate: $>120$ beats $/ \mathrm{min}$

- $\quad$ Oxygen saturation $<92 \%$ under $4 \mathrm{l} / \mathrm{min} 02$

Blood samples for blood counts (CBC) of patients with COVID-19 were taken on admission.

The control group had an RT-PCR for SARS CoV-2 which was negative.

Data entry was performed on Excel 2013, with a uni-variate descriptive method using percentages and averages. The software used for the $p$-value is epi info 7.2. A value of $p<0.05$ is considered statistically significant.

\section{Results}

\subsection{Hematopoietic profile of patients with COVID-19 at admission}

Of the 102 patients with Covid-19, most had abnormalities in peripheral blood parameters when compared to the control group.

Table 1 Blood counts in COVID 19 patients at admission and controls

\begin{tabular}{|l|l|l|l|}
\hline Variations & Covid-19 cases $(\mathbf{n}=102)$ & control cases $(n=38)$ & $P$ value \\
\hline Age & 43,73 & 42 & 0.5 \\
\hline Male vs female & $54,16 \%$ vs $45,83 \%$ & $52.8 \%$ vs $47.2 \%$ & 0.7 \\
\hline Leukocytes $\left(\times 10^{9} / \mathrm{L}\right)$ & $8.32(6.57-10.08)$ & $5.85(5.16-6.76)$ & $<0.001$ \\
\hline Neutrophils $\left(\times 10^{9} / \mathrm{L}\right)$ & $5,33(4,2-6,45)$ & $3.27(2.61-3.94)$ & $<0.001$ \\
\hline Lymphocytes $\left(\times 10^{9} / \mathrm{L}\right)$ & $2,02(1,58-2,45)$ & $2.15(1.83-2.34)$ & 0.29 \\
\hline Hemoglobin $(\mathrm{g} / \mathrm{dl})$ & $13,5(10,6-16,37)$ & $14,8(13,5-15,6)$ & $<0.001$ \\
\hline Platelets $\left(\times 10^{9} / \mathrm{L}\right)$ & $251(340-162)$ & $266(215,4-316,6)$ & 0.3 \\
\hline NLR & 2,63 & $1,52(1,42-1,68)$ & $<0.001$ \\
\hline PLR & 124,2 & $124(117,5-135)$ & 0.4 \\
\hline
\end{tabular}

WBCs in the patient group were $8.32 \times 109 / \mathrm{L}$ vs $5.85 \times 109 / \mathrm{L}, \mathrm{p}<0.001$, an elevated neutrophil count $(5.33 \times 109 / \mathrm{L}$ vs $3.27 \times 109 / \mathrm{L}, \mathrm{p}<0.001)$, a lower hemoglobin concentration $(13.5$ [10.6-16.37] g/dl vs 14.8 [13.5-15.6] g/dl, p<0.001), and a higher NLR (2.63 vs $1.52, \mathrm{p}<0.001)$.

There were no significant differences in age, sex ratio, lymphocyte and platelet counts between the patient and control groups (table 1). 
Severe cases had a higher white blood cell count $(14,279( \pm 6,564)$ vs. 7,471 $( \pm 2,609) \times 109 / \mathrm{L}, \mathrm{p}<0.001)$, a much higher neutrophil count $(12,547( \pm 6,698)$ vs. 4,468 $( \pm 1,882) \times 109 / \mathrm{L}, \mathrm{p}<0.001)$. Lymphopenia $(0.851( \pm 0.406)$ vs. 2.138 $( \pm 1.103) \times 109 / \mathrm{L}, \mathrm{p}<0.001)$, inflammatory anemia was noted $(11.1( \pm 2.4)$ vs. $13.6( \pm 2.7) \mathrm{g} / \mathrm{dl}, \mathrm{p}<0.001)$, NLR $(14.74$ vs. 2.08) and PLR (145 vs. 117) were higher than the non-severe COVID-19 cases. There was no significant difference in platelet levels between the two groups (Table 2).

Table 2 Blood counts in patients with non-severe and severe Covid-19 on admission

\begin{tabular}{|l|l|l|l|}
\hline Variations & $\begin{array}{l}\text { Mild to moderate Covid-19 } \\
\text { cases }\end{array}$ & Severe Covid-19 cases & P value \\
\hline Average age & $41,5( \pm 17.7)$ ans & $61,4( \pm 15.9)$ ans & $<0.001$ \\
\hline Leukocytes $\left(\times 10^{9} / \mathrm{L}\right)$ & $7.471( \pm 2.609)$ & $14.279( \pm 6.564)$ & $<0.001$ \\
\hline Neutrophils $\left(\times 10^{9} / \mathrm{L}\right)$ & $4.468( \pm 1.882)$ & $12.547( \pm 6.698)$ & $<0.001$ \\
\hline Lymphocytes $\left(\times 10^{9} / \mathrm{L}\right)$ & $2.138( \pm 1.103)$ & $0.851( \pm 0.406)$ & $<0.001$ \\
\hline Hemoglobin $(\mathrm{g} / \mathrm{dl})$ & $13,6( \pm 2.7)$ & $11,1( \pm 2.4)$ & $<0.001$ \\
\hline Platelets $\left(\times 10^{9} / \mathrm{L}\right)$ & $255( \pm 110)$ & $240( \pm 90)$ & 0.3 \\
\hline NLR & 2,08 & 14,74 & $<0.001$ \\
\hline PLR & 117 & 145 & $<0.001$ \\
\hline
\end{tabular}

\section{Discussion}

COVID-19 is a systemic disease causing multiple organ damage, caused by coronavirus 2 (SARS-CoV 2), with the lung as the main target organ. It can cause severe lung damage and acute respiratory distress syndrome (ARDS) in severe cases that can lead to death [3]. The virus binds to the ACE2 receptor and enters the alveolar epithelial cells [4], causing the cells to release inflammatory factors and activate the numerous macrophages in the alveolar tissue; the macrophages then induced factors and chemokines, which recruit large numbers of mononuclear immune cells to aggregate and infiltrate the lung tissue; this additional immune activation leads to a storm of inflammation and tissue damage [5].

The significant decrease in lymphocyte numbers in patients with COVID-19 may be related to the redistribution and increased consumption of lymphocytes and defective hematopoiesis [6]. Li et al. found that bacterial pneumonia is added to some deaths due to COVID-19 [9]. This explains the significant increase in leukocyte and neutrophil counts. It has been reported that SARS-CoV 2 causes damage to ACE2 receptor-rich kidney tissue and an increase in inflammatory factors, which can lead to reduced erythropoiesis and destruction of red blood cells, leading to anemia [8].

In our series, blood count parameters in patients with COVID-19 were significantly abnormal. Among them, increased white blood cell count and neutrophil counts, decreased lymphocyte count was most evident in severe Covid 19 cases. Hemoglobin was also significantly lower than that of the healthy control group. The combined peripheral blood parameters, including NLR was significantly increased. This indicates that monitoring changes in routine blood parameters had a important clinical significance. Studies have shown that the progression and prognosis of COVID-19 is related to the body's immune status and excessive inflammatory response [8]. In this study, by comparing and analyzing routine peripheral blood parameters of the Covid 19 in non-severe and severe groups, it was found that the absolute value of lymphocytes in the severe group was significantly lower than that of the non-severe group, while the number of inflammatory neutrophils in the severe group was much higher than that of the mild group. These changes showed that the degree of inflammation had further intensified, triggering an inflammatory storm and leading to increased tissue and cell damage [10]. Low lymphocyte levels and impaired immune cell function cause a dysfunction of the immune system, so patients with severe COVID-19 may be more susceptible to bacterial infection [11]. The NLR blood cell parameter reflects this situation, and we therefore believe that NLR has some value in judging the severity of COVID-19. Our results are consistent with those of Qin et al [12] 


\section{Conclusion}

In light of these data, we find that COVID-19 will cause abnormalities of the hematopoietic system. The most obvious abnormalities are lymphopenia, increased leukocyte count and NLR ratio, which are re-evaluated according to the severity of the disease and clinical classification. Thus, regular monitoring of blood counts should be involved to judge the progression and prognosis of COVID-19.

\section{Compliance with ethical standards}

\section{Acknowledgments}

We would like to thank our masters, specialists, residents, and nurses from the pneumology department of Marrakesh University Hospital for all the support they have provided during the preparation of this work.

\section{Disclosure of conflict of interest}

The authors declare no conflict of interest.

\section{Statement of informed consent}

Permission to conduct the study was obtained from Cadi Ayyad University, Department of Pneumology, ARRAZI Hospital, Mohamed VI University Hospital Center, Marrakesh. Informed consent was obtained from patients. All patients' information including raw data was be kept confidential during and after study period.

\section{References}

[1] Jan Hc, Wh Yang, Ch Ou. Combination of the preoperative systemic immune-inflammation index and monocytelymphocyte ratio as a novel prognostic factor in patients with upper-tract urothelial carcinoma, Ann. Surgical Oncol. 2019; 26: 669-684.

[2] T Demirdal, P Sen. The significance of neutrophil-lymphocyte ratio, platelet lymphocyte ratio and lymphocytemonocyte ratio in predicting peripheral arterial disease, peripheral neuropathy, osteomyelitis and amputation in diabetic foot infection, Diabetes Res. Clin. Pract. 2018; 144: 118-125.

[3] Guan Wj, HuY Ni Zy, et al., Clinical characteristics of coronavirus disease 2019 in China, New Engl. J. Med. 2020.

[4] Y Wan, J Shang, R Graham, et autres, Receptor recognition by the novel coronavirus from Wuhan : an analysis based on decade-long structural studies of SARS cor- onavirus, J. Virol. 2020; 94.

[5] C Huang, Y Wang, X Li, et al, Clinical features of patients infected with 2019, novel coronavirus in Wuhan China, Lancet (Londres, Angleterre). 2020; 395: 497-506.

[6] Xh Yao, Li Ty, He Zc, et autres, Un rapport pathologique de trois cas COVID-19 par autopsies minimalement invasives, Zhonghua bing li xue za zhi = J. Pathol chinois. 2020; 49: E009.

[7] Deng Yy, Y Zheng, Cai Gy, et al, Single-cell RNA sequencing data suggest a role for angiotensin-converting enzyme 2 in kidney impairment in patients infected with 2019-nCoV, Chin. Med. J. 2020.

[8] Z Xu, L Shi, Y Wang, et autres, Pathological findings of COVID-19 associated with acute respiratory distress syndrome, Lancet Respirat. Med. 2020.

[9] X Li, L Wang, S Yan, et al, Clinical characteristics of 25 death cases with COVID- 19 : a retrospective review of medical records in a single medical center, Wuhan, China, Int. J. Infect. Dis : IJID : Off. Publ. int. Soc. Infect. Dis. 2020.

[10] P Mo, Y Xing, Y Xiao, et al, Clinical characteristics of refractory COVID-19 pneumonia in Wuhan, China, Clin. Infect. Dis : Off. Infect. publ. Dis. Soc. Am. 2020.

[11] N. Chen, M. Zhou, X. Dong, et al, Epidemiological and clinical characteristics of 99 cas 2019 de nouvelle pneumonie à coronavirus à Wuhan, Chine : une étude descriptive, Lancet (Londres, Angleterre). 2020; 395: 507-513.

[12] C Qin, L Zhou, Z Hu, et al, Dysregulation of immune response in patients with COVID-19 in Wuhan, China, Clin. Infect. Dys. : Off. Publ. Infect. Dis. Soc. Am. 2020. 